\title{
Effet des produits chimiques et des produits pharmaceutiques sur la spermatogenèse
}

\author{
J.A. THOMAS \\ Department of Pharmacology, University of Texas Health Science Center, San Antonio, TX \\ 78284, U.S.A.
}

Il y a eu ces dernières années une discussion continue sur le déclin des numérations de spermatozoïdes. La discussion s'est principalement centrée sur la mise en évidence d'une relation cause-effet. Il n'y a eu aucune absence d'hypothèses en ce qui concerne les causes qui étaient responsables de ce prétendu déclin des numérations de sperme.

Alors que de nombreux facteurs, à la fois chimiques et non chimiques, peuvent affecter la spermatogenèse, le débat actuel sur la diminution de la qualité du sperme durant les 50 dernières années est né d'une revue des publications sélectionnées à partir du Cumulated Index Medicus and Current List (19301965) et de la base de données du MEDLINE Silver Platter (1966-Aout 1991). Carlsen et al., ont rapporté qu'il y avait un authentique déclin de la qualité du sperme sur les 50 dernières années. Ces auteurs ont aussi mis en évidence une augmentation de l'incidence des anomalies uro-génitales incluant le cancer du testicule, la cryptorchidie et l'hypospadias. Les résultats de Carlsen et al., ont été ré-analysés et mis en doute par Bromwich et al. Carlsen et al., utilisèrent une analyse par régression linéaire de 61 jeux différents de données obtenues entre 1938 et 1990. La ré-analyse de ces 61 études révéla que pour 48 de ces études publiées depuis 1970 , et qui comptaient pour $88 \%,(13217)$ des hommes étudiés, il y avait en fait une augmentation des numérations de sperme. Plusieurs facteurs furent inclus dans cette ré-analyse comprenant des modifications des plus basses valeurs de référence, du poids statistique inapproprié de certaines des études de plus petite taille, et l'utilisation de modèles statistiques différents. C'est pourquoi la preuve de départ, qui utilisait une méta-analyse ne soutient plus l'hypothèse que la numération de sperme ait diminué significativement entre 1940 et 1990.

Shalpe et Sakkebaek ont avance l'hypothèse que l'augmentation de l'incidence des anomalies repoductives chez l'homme pouvait être liée à une augmentation de l'exposition aux estrogènes in utero. Leur hypothèse inclut une augmentation de l'exposition au diethylstilbestrol (DES) et la présence possible des œstrogènes environnementaux (ex. DDT). Il est aussi possible que les estrogènes et les antiestrogènes de l'alimentation et de l'environnement puissent exercer des actions contraires et ces interactions doivent être prises en considération dans l'évaluation globale du risque des impacts négatifs potentiels sur la santé humaine et environnementale représenté par ces substances chimiques.

Auger et al. tendent à corroborer les résultats de Carlsen et al. en rapportant un déclin de la qualité du sperme sur les 20 dernières années chez les hommes féconds de la région parisienne. Alors que ces auteurs ne rapportent aucune modification du volume de l'éjaculat, la concentration moyenne de sperme a diminué d'environ $2 \% /$ an (de 89 x $106 / \mathrm{ml}$ en 1973 à $60 \times 10^{6} / \mathrm{ml}$ en 1992). 